\title{
COMPRESSIBLE MATRIX RINGS
}

\section{Efraim P. ARMEndariz AND JAE KeOL PARK}

Let $Z(K)$ denote the center of a ring $K$. A ring $R$ is compressible if $Z(e R e)=e Z(R)$ for each idempotent $e$ of $R$. In response to a question of $\mathrm{S}$. Berberian, G. Bergman has constructed a (non-commutative) integral domain, satisfying a polynomial identity, for which the $2 \times 2$ matrix ring over the domain is not compressible. In contrast to Bergman's example, we show that the ring of $n \times n$ matrices over any commutative ring is always compressible.

The example constructed by Bergman [3] eliminates a large class of rings, prime Goldie rings, for which compressibility is a Morita invariant property. In a positive vein, A. Page has shown in unpublished work that compressibility is preserved in matrix rings over (compressible) von Neumann regular rings.

In contrast to Bergman's P.I. domain we have been able to show that $R_{n}$ is always compressible whenever $R$ is a commutative integral domain. In fact, a much more general result holds.

THEOREM 1. If $R$ is a commutative ring then $R_{n}$ is compressible for all $n \geq 1$.

Proof. Let $A=R_{n}$ and $\varepsilon \in A$ with $\varepsilon^{2}=\varepsilon$. From Passman [4, Lemma 4.5, p. 253] we have $A \varepsilon A \cap \ell_{A}(A \varepsilon A)=0$. We claim that

Received 27 April 1984.

Copyright Clearance Centre, Inc. Serial-fee code: 0004-9727/84 $\$ \mathrm{~A} 2.00+0.00$ 
$A=A \varepsilon A \oplus \ell_{A}(A \varepsilon A)$. Indeed $\varepsilon\left(I_{n}-\varepsilon\right)=0$ so we have $\varepsilon\left(I_{n}-\varepsilon\right) \operatorname{adj}\left(I_{n}-\varepsilon\right)=0$. However $\left(I_{n}-\varepsilon\right) \operatorname{adj}\left(I_{n}-\varepsilon\right)=\operatorname{det}\left(I_{n}-\varepsilon\right) I_{n}$. Hence $\operatorname{det}\left(I_{n}-\varepsilon\right) \varepsilon=\varepsilon \cdot \operatorname{det}\left(I_{n}-\varepsilon\right) I_{n}=0$. Let $U$ be the ideal of $R$ senerated by the entries of $\varepsilon$. Then $\operatorname{det}\left(I_{n}-\varepsilon\right)=1-x$ where $x \in U$. Since $(1-x) \varepsilon=0$ and $(1-x) I_{n} \in Z(A)$ we have $(1-x) I_{n} \in \ell_{A}(A \varepsilon A)$. Thus $I_{n}-x I_{n} \in \ell_{A}(A \in A)$. Because $A \varepsilon A$ contains all matrices $y I_{n}$ with $y \in U$ we have $x I_{n} \in A \in A$ and so $I_{n} \in A \varepsilon A+\ell_{A}(A \varepsilon A)$. According'y $A=A \varepsilon A \oplus \ell_{A}(A \in A)$ as we claimed. However, both $A \varepsilon A$ and ${ }_{A}(A \in)$ are ideals of $A$ so $A \varepsilon A=A \varphi$ for some central idempotent $\varphi \in A$. risen $\varepsilon:=\varepsilon \varphi$ so $\varepsilon A \varepsilon=\varepsilon A \varphi \varepsilon$; hence we may assume that $A=A \varepsilon A$. Now suppose $x \in Z(\varepsilon A \varepsilon)$. The mapping $\theta: A \rightarrow A$ given by $\theta\left(\sum \alpha_{i} \varepsilon \beta_{i}\right)=\sum \alpha_{i} x \beta_{i}$ is well-defined. Indeed, if $\sum \alpha_{i} \varepsilon \beta_{i}=0$ then for all $\alpha, \beta \in A$ we have $\left(\sum_{i} \alpha_{i} x \beta_{i}\right) \alpha \varepsilon \beta=\sum_{i} \alpha_{i} x\left(\varepsilon \beta_{i} \alpha \varepsilon\right) \beta=\sum_{i} \alpha_{i} \varepsilon \beta_{i} \alpha x \beta=$ $\left(\sum_{i} \alpha_{i} \varepsilon B_{i}\right) \alpha x \beta=0$; therefore $\left[\alpha_{i} x B_{i} \in \ell_{A}(A)=0\right.$ showing that $\theta$ is wclldefined. Then because $x \in Z(\varepsilon A \varepsilon), \theta$ is an $A-A$-bimodule mapping. Thus $\theta$ is given by a right multiplication by a central element $z$ of $A$. In particular, $x=\theta(\varepsilon)=\varepsilon z$ and so $x \in \varepsilon Z(A)$. Since we always have $E Z(A) \subseteq Z(\varepsilon A \varepsilon)$ the proof is complete.

An immediate consequence is the

COROLLARY. If $M$ is a finitely generated projective R-module over a commutative ring $R$ then $\operatorname{Hom}_{R}(M, M)$ is a compressible ring.

Recall that an algebra $A$ over a commutative ring $R$ is a separable $R$-algebra in case $A$ is a projective $A^{e}$-module, where $A^{e}=A \otimes_{R} A^{O p}$ is the enveloping algebra of $A$. The separable $R$-algebra $A$ is central separable, or an Azumaya algebra if $R=Z(A)$. (See [3] for properties of separable algebras.)

THEOREM 2. An Azrmaya algebra $A$ over a commutative ring $R$ is compressible.

Proof. Since $A$ is Azumaya we have $A \cong \operatorname{Hom}_{R}(A, A)$ and $R^{A}$ is a progenerator. By the corollary, $A^{e}$ is a compressible ring. Furthermore $R$ is both a left and right $R$-module direct summand of $A$. Suppose $f$ is 
an idempotent in $A$. Then $f \otimes 1$ is an idempotent in $A^{e}$ and $Z\left((f \otimes 1) A^{e}(f \otimes 1)\right)=(f \otimes 1) Z\left(A^{e}\right)$. Also $Z(f A f) \bigotimes_{R} R \subseteq f A f \otimes_{R} R$. Since $R$ is a direct summand of $R^{A}$ we also have that $R$ is a direct summand of $A^{A^{O P}}$ and so $f A f \otimes_{R} R \subseteq f A f \otimes_{R} A^{O P}$. However every element of $Z(f A f) \otimes_{R} R$ is central in $f A f \otimes_{R} A^{o p}$ so that, in fact,

$$
\begin{aligned}
Z(f A f) \bigotimes_{R} R & \subseteq Z\left(f A f \otimes_{R} A^{O P}\right) \\
& =Z\left((f \otimes 1) A^{e}(f \otimes 1)\right)=(f \otimes 1) Z\left(A^{e}\right) .
\end{aligned}
$$

If $t \in Z(f A f)$ then $t \otimes 1=(f \otimes 1)\left(\Sigma a_{i} \otimes b_{i}\right)=\Sigma f a_{i} \otimes b_{i}$, where $\Sigma a_{i} \otimes b_{i} \in Z\left(A^{e}\right)$. Now the isomorphism $\theta: A^{e} \rightarrow \operatorname{Hom}_{R}(A, A)$ is given by $\theta\left(\Sigma c_{i} \otimes d_{i}\right): a \rightarrow \Sigma c_{i} a d_{i}$. Thus $\theta\left(\Sigma a_{i} \otimes b_{i}\right) \in Z\left(\operatorname{Hom}_{R}(A, A)\right)$ hence $\theta\left(\Sigma a_{i} \otimes b_{i}\right)$ commutes with all left and right multiplications by elements of $A$. Let $a_{\lambda}, a_{\rho}$ denote left and right multiplication by $a \in A$, respectively. Then we have $\left(\theta\left(\Sigma a_{i} \otimes b_{i}\right) \circ a_{\lambda}\right)(1)=\theta\left(\Sigma a_{i} \otimes b_{i}\right)(a)=$ $\Sigma a_{i} a b_{i}$ and $\left(a_{\lambda} \circ \theta\left(\Sigma a_{i} \otimes b_{i}\right)\right)(1)=a \Sigma a_{i} b_{i}$; thus $\Sigma a_{i} a b_{i}=a\left(\Sigma a_{0} b_{i}\right)$. Using $a_{\rho}$ we also have $\Sigma a_{i} a b_{i}=\left(\Sigma a_{i} b_{i}\right) a$. Combining these we get $a\left(\Sigma a_{i} b_{i}\right)=\left(\Sigma a_{i} b_{i}\right) a$ for all $a \in A$. Therefore $\Sigma a_{i} b_{i} \in Z(A)=R$. From $t \otimes 1=\Sigma f a_{i} \otimes b_{i}$ with $\sum a_{i} \otimes b_{i} \in Z\left(A^{e}\right)$, we have $\theta(t \otimes 1)(x)=t x$ and $\theta(t \otimes 1)(x)=\theta\left(\Sigma f a_{i} \otimes b_{i}\right)(x)=\Sigma f a_{i} x b_{i}$ for all $x \in A$.

In particular for $x=1$ we obtain $t=f\left(\Sigma a_{i} b_{i}\right) \in f Z(A)$. We conclude that $A$ is compressible.

By [3, Theorem 3.8], $A$ is a separable algebra over a commutative ring $R$ if and only if $A$ is central separable over $Z(A)$ and $Z(A)$ is a separable algebra over $R$. In particular $A$ is an Azumaya algebra over its center which yields:

COROLLARY. If $R$ is a commutative ring then any separable $R$-algebra is compressible.

A particular example of separability occurs when $G$ is a finite group whose order is a unit in the commutative ring $R$. Thus we have 
COROLLARY. If $R$ is a commutative ring, $G$ a finite group whose order is invertible in $R$ then the group ring $R G$ is compressible.

In a personal correspondence to the second author, K. Motose has pointed out that the group algebra $Z_{2}\left[S_{4}\right]$ is not a compressible $r i n g$. This example, coupled with Bergman's example, demonstrates the limitations of compressibility.

\section{REFERENCES}

[1] S. K. Berberian, "The center of a corner of a ring", J. Algebra 71 (1981), 515-523.

[2] G. M. Bergman, "Some examples of non-compressible rings", Comm. in Algebra 12 (1984), 1-8.

[3] F. DeMeyer and E. C. Ingraham, Separable algebras over commitative rings (Lecture Notes in Mathematics, Vol. 181. Springer-Verlag, New York and Berlin, 1971).

[4] D. S. Passman, The algebraic stmucture of group rings (John Wiley, New York, 1977).

Department of Mathematics,

University of Texas,

Austin,

Texas 78712 ,

U.S.A.

Department of Mathematics,

Busan National University,

Busan 607,

Korea . 